\title{
Annealing effect on photoacoustic characterization of NiSe metal chalcogenide semiconductor using phase signal analysis
}

\begin{abstract}
Nickel selenide (NiSe) has been synthesized by solid state method and annealed at five different temperatures, ranging from $323 \mathrm{~K}$ to $823 \mathrm{~K}$. The annealing effect on NiSe thermal and carrier transport properties were investigated by using open-cell photoacoustic technique. From analysis of its phase signal-frequency, thermal diffusivity, carrier diffusion coefficient, surface recombination velocity and recombination lifetime of the NiSe was determined. The results show that with increasing of the annealing temperature of NiSe sample, the thermal diffusivity and the carrier diffusion coefficient increased. The surface recombination velocity was decreasing as the annealing temperature of the sample increased. The increasing of annealing temperature of the sample also affected the trend of band-to-band recombination lifetime.
\end{abstract}

Keyword: Nickel selenide; Photoacoustic 